\title{
Foreign Body Response and Intravital Microscopy on AIW in Mice
}

\author{
Shannen K. Sharpe ${ }^{1}$, Michelle M. Martinez ${ }^{2}$, Kenneth W. Dunn² \\ 1.Indiana University School of Medicine;, Indiana University Department of Medicine, \\ Division of Nephrology
}

The foreign body response is the body's response to the insertion of an object. The foreign body response consists of two components, the innate and adaptive immune response, and lasts for the life of the inserted object. Accordingly, the foreign body response represents a significant challenge to the development of implanted medical devices. In addition to triggering the damaging consequences of inflammation, the foreign body response acts to encapsulate and isolate inserted objects, limiting the functional lifetime of medical devices such as glucose monitors. Accordingly, significant efforts have been devoted to understanding the cell biology of the foreign body response to identify approaches for limiting surface "biofouling". We have developed an indwelling window system that support longitudinal intravital microscopy of mice. In studies of transgenic mice expressing fluorescent immune cells, we found that the window triggers a local inflammatory response. To explore the utility of this window system as an experimental platform for characterizing the foreign body response, we conducted an intravital microscopy study of 8 mice expressing GFP in myeloid immune cells (Lys-EGFP mice) with surgically implanted abdominal imaging windows. To identify differences in the responses to different surface chemistries, the windows were either left uncoated or coated with poly-L-lysine or type $\mathrm{V}$ mouse collagen prior to insertion. Intravital multiphoton microscopy studies conducted over a period of up to 3 weeks demonstrated that the window instigated a local recruitment of immune cells, followed by vascularization and giant cell formation that varied depending upon window surface treatment. These studies demonstrate the utility of the abdominal window as a model system for studying the cell biology of the foreign body response and represent the template for subsequent studies designed to compare the foreign body response to different coating materials designed to extend the useful lifetime of implanted devices. 